\title{
Chronic immune checkpoint inhibitor pneumonitis
}

To cite: Naidoo J, Cottrell TR, Lipson EJ, et al. Chronic immune checkpoint inhibitor pneumonitis. Journal for ImmunoTherapy of Cancer 2020;8:e000840. doi:10.1136/ jitc-2020-000840

- Additional material is published online only. To view please visit the journal online (http://dx.doi.org/10.1136/jitc2020-000840).

Accepted 07 May 2020

Check for updates

(C) Author(s) (or their employer(s)) 2020. Re-use permitted under CC BY-NC. No commercial re-use. See rights and permissions. Published by BMJ.

For numbered affiliations see end of article.

Correspondence to Dr Jarushka Naidoo jnaid001@jhmi.edu

Jarushka Naidoo (1) , ${ }^{1,2}$ Tricia R Cottrell, ${ }^{3}$ Evan J Lipson (10 , ${ }^{1,2}$ Patrick M Forde, ${ }^{1,2}$ Peter B Illei, ${ }^{4}$ Lonny B Yarmus, ${ }^{5}$ K Ranh Voong, ${ }^{6}$ David Feller-Kopman, ${ }^{5}$ Hans Lee, ${ }^{5}$ Joanne Riemer, ${ }^{1}$ Daphne Wang, ${ }^{4}$ Janis M Taube ${ }^{4}$ Julie R Brahmer ${ }^{1,2}$ Cheng Ting Lin, ${ }^{7}$ Sonye K Danoff, ${ }^{5}$ Franco R D'Alessio, ${ }^{5}$ Karthik Suresh ${ }^{5}$

\section{ABSTRACT}

Background Pneumonitis from immune checkpoint inhibitors (ICI) is a potentially fatal immune-related adverse event (irAE) from antiprogrammed death 1/ programmed death ligand 1 immunotherapy. Most cases of ICl pneumonitis improve or resolve with 4-6 weeks of corticosteroid therapy. Herein, we report the incidence, clinicopathological features and management of patients with non-small cell lungcancer (NSCLC) and melanoma who developed chronic ICI pneumonitis that warrants $\geq 12$ weeks of immunosuppression.

Methods Patients with ICl pneumonitis were identified from institutional databases of ICl-treated patients with advanced melanoma and NSCLC between January 2011 and July 2018. ICI pneumonitis was defined as clinical/ radiographic evidence of lung inflammation without alternative diagnoses, adjudicated by a multidisciplinary team. Chronic ICI pneumonitis was defined as pneumonitis that persists or worsens with steroid tapering, and necessitates $\geq 12$ weeks of immunosuppression, after $\mathrm{ICl}$ discontinuation. Serial chest CT was used to assess radiological features, and tumor response by Response EvaluationCriteria for Solid Tumors V.1.1. Bronchoalveolar lavage fluid (BALF) samples were assessed by cell differential. Lung biopsy samples were evaluated by H\&E staining and multiplex immunofluorescence $(\mathrm{mIF})$, where available.

Results Among 299 patients, 44 developed ICl pneumonitis (NSCLC: 5/205; melanoma: 1/94), and of these, 6 experienced chronic ICI pneumonitis. The overall incidence of chronic ICl pneumonitis was thus $2 \%$. Of those who developed chronic ICI pneumonitis: the majority had NSCLC (5/6), all sustained disease control from ICls, and none had other concurrent irAEs. Timing of chronic ICI pneumonitis development was variable (range: $0-50$ months), and occurred at a median of 12 months post ICI start. Recrudescence of ICl pneumonitis occurred at a median of 6 weeks after initial steroid start (range: 3-12 weeks), with all patients requiring steroid reintroduction when tapered to $\leq 10 \mathrm{mg}$ prednisone/equivalent. The median total duration of steroids was 37 weeks (range: 16-43+weeks). Re-emergence of radiographic ICI pneumonitis occurred in the same locations on chest CT, in most cases (5/6). All patients who developed chronic ICI pneumonitis had BALF lymphocytosis on cell differential and organising pneumonia on lung biopsy at initial ICI pneumonitis presentation, with persistent BALF lymphocytosis and brisk CD8+ infiltration on $\mathrm{mIF}$ at pneumonitis re-emergence during steroid taper.
Conclusions A subset of patients who develop pneumonitis from ICls will develop chronic ICI pneumonitis, that warrants long-term immunosuppression of $\geq 12$ weeks, and has distinct clinicopathological features.

\section{INTRODUCTION}

Pneumonitis is an uncommon but potentially fatal toxicity of anti-PD(L) 1 immune checkpoint inhibitors (ICI) for cancer. ${ }^{1-3}$ The incidence of this toxicity is approximately $5 \%$ in patients with solid tumors treated with anti$\mathrm{PD}(\mathrm{L}) 1$ monotherapy, and up to $10 \%$, in patients receiving anti-PD(L) 1-based combinations such as ipilimumab/nivolumab, or those with non-small cell lung cancer (NSCLC). ${ }^{4}$ Most cases of ICI pneumonitis resolve or improve with corticosteroids; however, a small proportion of patients may develop recurrent pneumonitis when rechallenged with ICIs. ${ }^{4}$ Distinct from this phenomenon, we recently observed a clinical phenotype of 'chronic ICI pneumonitis', where patients exhibit persistent pneumonitis without immunotherapy rechallenge. Specifically, we observed clinical and radiographic evidence of ICI pneumonitis with exacerbation of symptoms when steroids were downtitrated, despite prompt discontinuation of ICI therapy at symptom onset, necessitating an extended course of immunosuppression to $\geq 12$ weeks. This duration of corticosteroids for ICI pneumonitis is well beyond the published guideline recommendations of 4-6 weeks. ${ }^{6-8}$ Since the features of this previously unreported clinical entity are unknown, we report herein the incidence, clinical presentation, radiographic, pathological features and management of patients who developed chronic ICI pneumonitis.

\section{METHODS}

\section{Patient selection}

We retrospectively identified patients with advanced NSCLC or melanoma treated with anti-PD(L)1 ICIs at Johns Hopkins Hospital 
between January 2011 and July 2018, who were enrolled on institutional biospecimen collection protocols. Patients may have received any anti-PD $(\mathrm{L}) 1$ agent either as standard of care or part of a clinical trial. Follow-up data were available for all patients through December 2018.

\section{Chronic ICI pneumonitis definitions and diagnosis}

The diagnosis of ICI pneumonitis was determined by the treating medical oncologist and confirmed by a multidisciplinary team, comprising a radiologist, pulmonologist, pathologist, and second medical oncologist. Pneumonitis was defined as clinical and radiographic evidence of lung inflammation after anti-PD $(\mathrm{L}) 1$ therapy, where alternative diagnoses such as confirmed infection and progressive cancer had been ruled out and multidisciplinary consensus had been reached. Chronic ICI pneumonitis was defined as clinical and radiographic evidence of pneumonitis that either (1) persisted at the end of recommend steroid tapering guidelines (4-6 weeks) $)^{6-8}$ or (2) worsened during steroid tapering warranting increased steroid dosing and/ or additional immunosuppression, and (3) necessitated a total duration of immunosuppression of $\geq 12$ weeks. ${ }^{6}$ The decision to repeat bronchoscopy with evaluation of bronchoalveolar lavage fluid (BALF) samples for a symptomatic patient with ICI pneumonitis during steroid tapering was based on: worsening dyspnea, persistent exertional desaturation, ongoing supplemental oxygen requirement, and absence of heart failure or anemia. In addition to bronchoscopy with BALF acquisition to rule out infection, any new areas of mass-like consolidation were biopsied to rule out tumor progression in relevant cases. Resolution of ICI pneumonitis was defined as complete weaning off steroids followed by no evidence of new lung abnormalities on chest CT or worsening dyspnea for at least 3 months.

\section{Radiology}

Serial radiological imaging for ICI pneumonitis with chest CT was collected, and tumor radiological response by Response Evaluation Criteria for Solid Tumors (RECIST) 1.1 (v. 4.03) was reported.

\section{Pathology}

BALF samples were assessed by automated cell differential. Lung biopsy samples where available were pathologically assessed by a thoracic pathologist using H\&E staining. Profiling of the inflammatory microenvironment with immunofluorescence (mIF) was completed in available samples, as previously described. ${ }^{9}$ Briefly, $4 \mu \mathrm{m}$ thick formalin-fixed, paraffin-embedded sections were stained with a 6-plex panel, including Ki67, CD8, CD20, programmed death 1 (PD-1), Pan-cytokeratin, and CD4 (online supplementary table 1 ).

\section{RESULTS}

\section{Incidence of chronic ICI pneumonitis}

We identified 205 patients with advanced NSCLC and 94 patients with advanced melanoma treated with anti-PD-1/ PD-L1 ICIs from January 2011 to July 2018. Among 299 patients, 44 developed ICI pneumonitis, and of these, 6 experienced chronic ICI pneumonitis. The overall incidence of chronic ICI pneumonitis in patients with advanced NSCLC and melanoma was 2\% (6/299), and $14 \%$ in patients who developed ICI pneumonitis $(6 / 44)$. In patients with NSCLC, $19 \%$ developed ICI pneumonitis $\left(39 / 205\right.$, reported elsewhere).$^{10}{ }^{11}$ In our original dataset of 39 pneumonitis cases in NSCLC, 4 developed chronic pneumonitis. Since that publication, we adjudicated one additional case of chronic pneumonitis, for a total of five cases in those with NSCLC. In patients with melanoma, 5\% developed ICI pneumonitis (5/94), and one patient developed chronic ICI pneumonitis. In total, six patients were identified as having developed chronic ICI pneumonitis, and the clinical features of this cohort are detailed below. Of note, chronic ICI pneumonitis eventually resolved with continued immunosuppression (either steroids alone or steroids in combination with a second immunosuppressive agent) in only three out of six patients.

\section{Clinical presentation of chronic ICI pneumonitis Baseline clinical characteristics}

The patient-level clinical details of patients who developed chronic ICI pneumonitis are depicted in table 1. The median age at time of diagnosis was 75 years (range: $56-77$ years). Five of the six patients were diagnosed with NSCLC as the primary tumor; one patient had melanoma. Only two of the six patients had received antecedent chemotherapy, and one of the six patients had a distant history of prior chest radiation. All patients demonstrated either a radiographic response or stable disease from immunotherapy.

\section{Comparison of chronic and acute ICl pneumonitis}

To contrast clinical characteristics of chronic ICI pneumonitis with cases of non-chronic (ie, acute) ICI pneumonitis, we compared the six cases of chronic ICI pneumonitis described here with 41 cases of acute ICI pneumonitis cases in NSCLC and melanoma (online supplementary table 2). While no differences in age, sex, tumor stage or histology, we did observe an increased proportion of combination ICI therapy in the chronic ICI pneumonitis group (83\%) compared with the acute ICI pneumonitis group (19\%; $\mathrm{p}=0.02)$. While the distribution of tumor histology subtypes was not significantly different between the groups, interestingly, both cases of adenocarcinoma in the chronic ICI pneumonitis group harbored KRASmutations.

\section{Timing of chronic ICI pneumonitis}

The first episode of pneumonitis in these patients occurred at a median time of 12 months after antiPD(L) 1 start (range: 0-50 months). One patient (patient 4) developed pneumonitis within a few hours of ICI infusion (0 days). In the other five patients, the median time to pneumonitis onset from ICI initiation was 12 months. Despite experiencing repeated bouts of ICI pneumonitis 
Table 1 Patients with chronic immune checkpoint inhibitor (ICl) pneumonitis

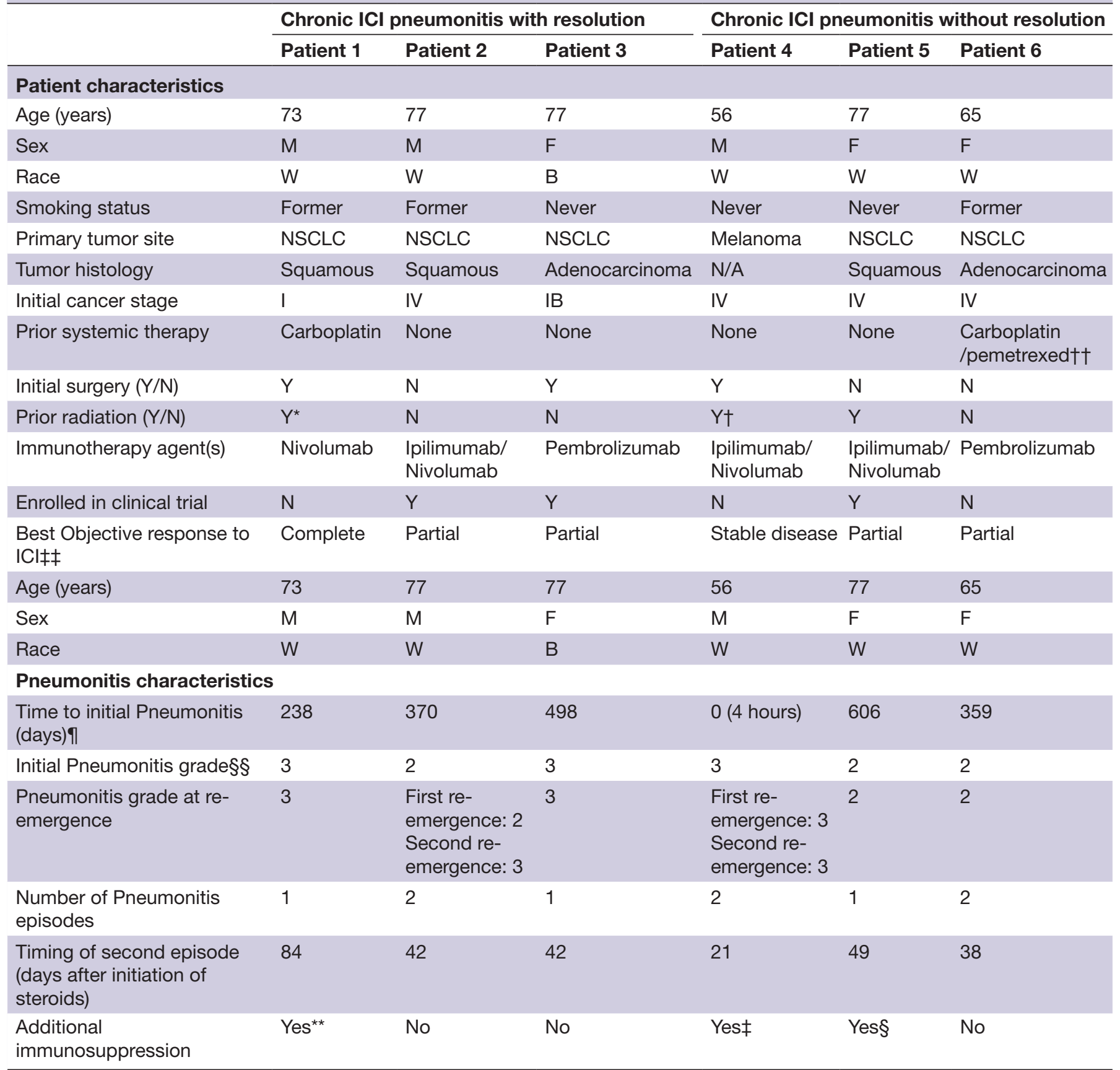

${ }^{*}$ Radiation: patient 1 received thoracic radiation for a lung metastasis 3 years prior to ICI start.

†Patient 4 received whole brain radiation for brain metastases 5 months after $\mathrm{ICI}$ start.

†Patient 4 not given a second course of steroids, started on infliximab, followed by intravenousimmunoglobulin (approximately 3 months after initiation of steroids).

§Patient 5 started a second course of steroids for ongoing symptoms (6 months after initiation of steroids) as well as starting mycophenolate.

qDay $0=$ start of $\mathrm{ICl}$.

${ }^{* *}$ Patient 1 treated with mycophenolate.

††Patient 6: received concurrent chemotherapy plus pembrolizumab for first four cycles.

‡¥Response to ICI by Response EvaluationCriteria for Solid Tumors V.1.1 criteria.

$\S \S C T C A E$ grade.

B, black; CTCAE, common toxicity criteria for adverse events; Ipi, ipilimumab; N/A, not applicable; Nivo, nivolumab; NSCLC, non-small cell lung cancer; W, Caucasian. 


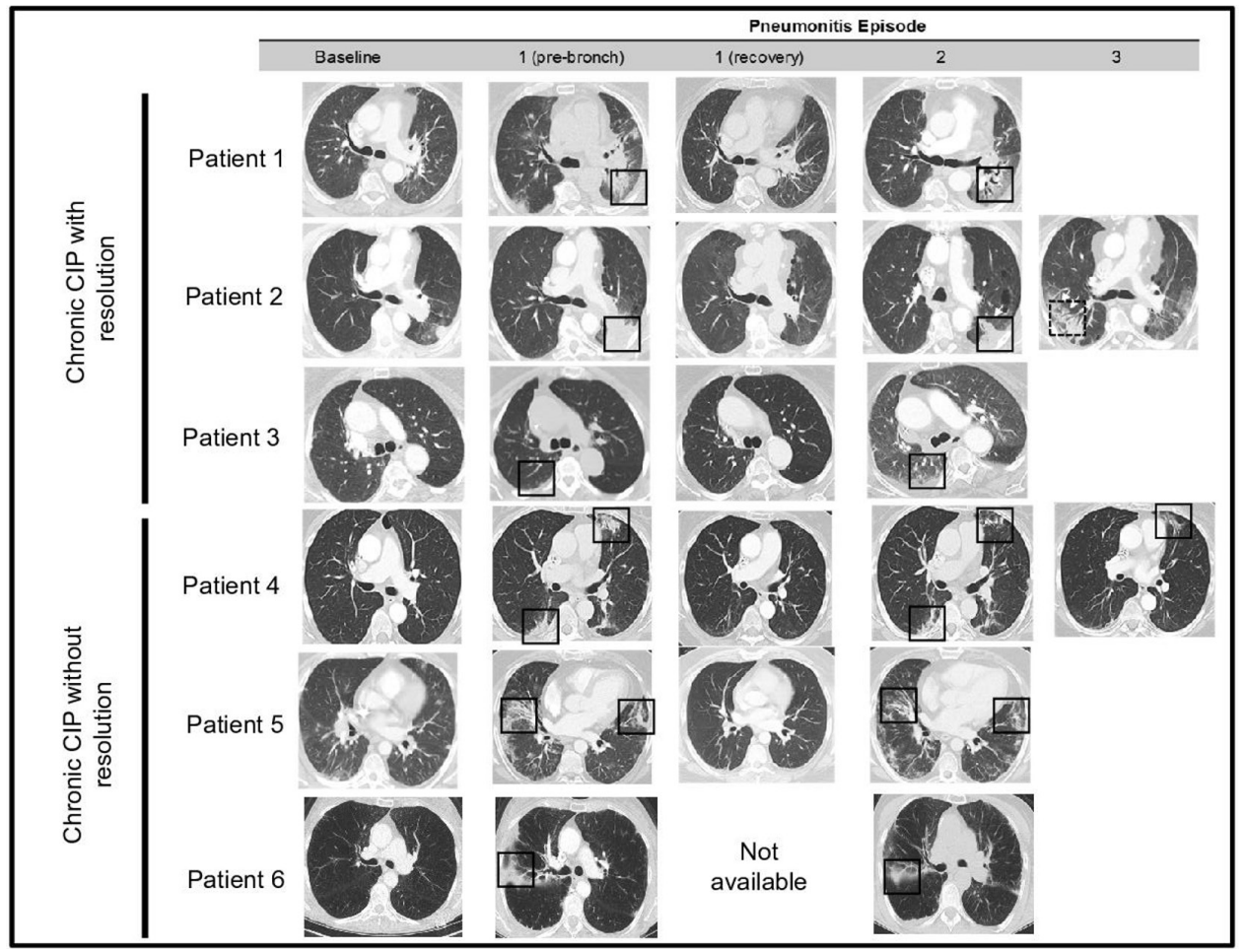

Figure 1 Serial radiological imaging in patients with chronic immune checkpoint inhibitor pneumonitis. Representative CT images at baseline-1: (pre-bronch): prior to first bronchoscopy, 1 (recovery): after treatment of first pneumonitis episode, 2: second episode of pneumonitis, 3: third episode of pneumonitis. Solid boxes show initial sites of pneumonitis and recrudescence, with radiographic pattern in keeping with acute lung injury or organizing pneumonia. Dotted line box highlights a new area of airspace opacity.

during steroid taper, the initial pneumonitis grade by CTCAE criteria was either 2 or 3 for all patients. No cases of grade 4 or grade 5 ICI pneumonitis were observed. All patients had been on steroids for at least 3 weeks when recrudescence of ICI pneumonitis was diagnosed, and the grade of ICI pneumonitis was also grade 3 or lower at these times. The second episode of ICI pneumonitis occurred at a median time of 6 weeks (range: 3-12 weeks) after the first episode. The grade of the subsequent bout of ICI pneumonitis was never higher than the initial event grade.

\section{Association with other irAEs}

While three of the six patients were also diagnosed with other non-pulmonary irAEs, none of the patients were diagnosed with irAEs concomitantly with ICI pneumonitis.

\section{Radiographic manifestations}

While the heterogeneity of radiographic findings in ICI pneumonitis has been described previously, the evolution of radiographic abnormalities on chest CT in patients with ICI pneumonitis who experience persistent and/ or repeated symptoms is not known. Thus, we examined serial CT radiography in this cohort of patients with chronic ICI pneumonitis. All patients underwent serial CT imaging to characterize radiographic abnormalities on chest CT during initial and subsequent presentations (figure 1). When ICI pneumonitis re-developed, we observed recrudescence of ground-glass opacities (GGO) or consolidations in the peribronchovascular distribution seen during the initial presentation (square boxes, figure 1). The pattern (GGO vs consolidation) of ICI pneumonitis abnormalities on chest CT was similar to the initial episode in five out of six instances. In one patient (patient 2), ICI pneumonitis re-emergence involved new airspace opacities in previously uninvolved portions of the lung parenchyma.

\section{Bronchoalveolar lavage findings}

While the BALF cell characteristics of ICI pneumonitis at initial presentation have been described by us previously, ${ }^{12}$ changes in the composition of the BALF cell differential in individual patients with repeated ICI pneumonitis episodes are unknown. In this cohort, all six patients underwent BALF and cell differential assessment at the time of initial ICI pneumonitis development and at re-emergence during steroid taper. Since only two patients experienced a third episode of ICI pneumonitis, we restricted our analyses to changes in BALF cell composition between the first and second ICI pneumonitis episode. Similar to our prior findings, the BALF differential was notable for significant lymphocytosis (approximately 30\%) at the initial ICI pneumonitis episode. Despite being treated with steroids, persistent (ie, >10\%) lymphocytosis was present in all six cases. Further, in four of the six cases, a higher lymphocyte percent was 


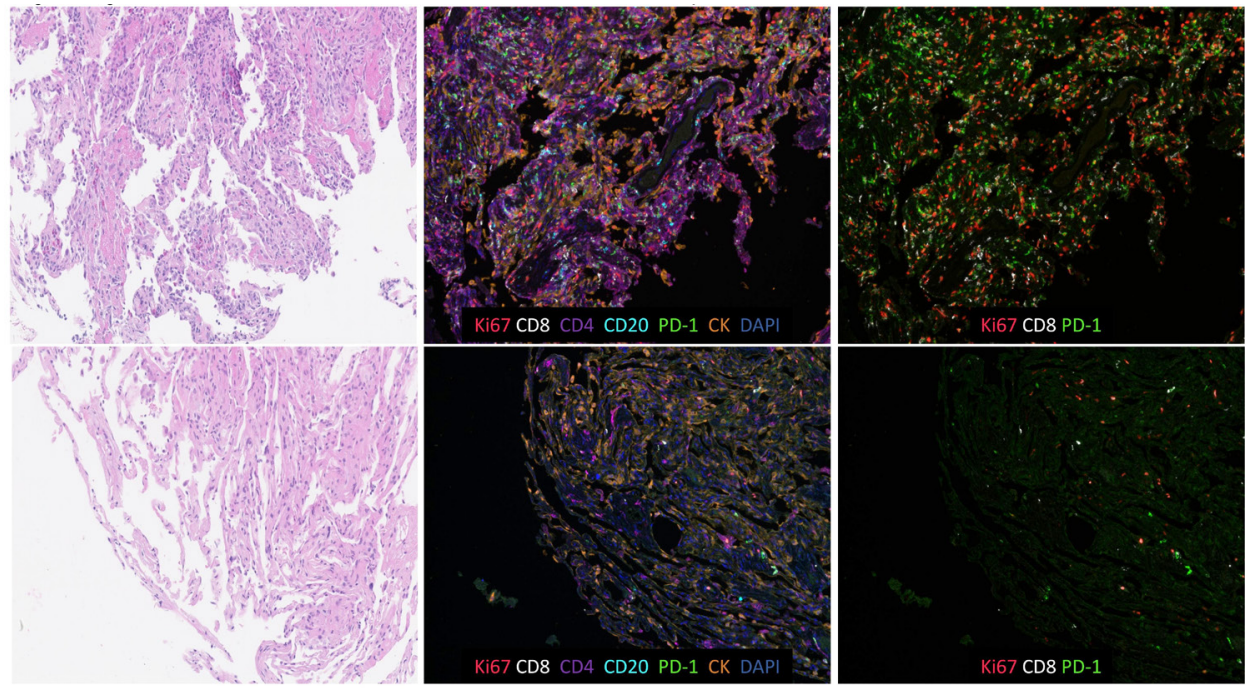

Figure 2 Pathological assessment by H\&E staining in chronic immune checkpoint inhibitor pneumonitis. (A) Patient 1: bronchiolitis obliterans organizing pneumonia (BOOP), described in manuscript as organizing pneumonia, with an intra-alveolar fibroblast plug and organizing alveolar fibrin. (B) Patient 2: acute lung injury with acute fibrinous organizing pneumonia and organizing diffuse alveolar damage. (C) Patient 3: organizing alveolar fibrin consistent with exudative phase of BOOP. (D) Patient 4: organizing pneumonia (BOOP) with organizing alveolar fibrin. (E) Patient 5: organizing alveolar fibrin consistent with exudative phase of BOOP. (F) Patient 6: organizing pneumonia (BOOP). PD-1, programmed death 1.

observed in the BALF obtained during the subsequent ICI pneumonitis presentation (online supplementary figure 1). In comparison, the percentage of monocytes remained either unchanged or decreased. The neutrophil percentage was $<50 \%$ in five out of six patients both on initial and repeat bronchoscopy.

\section{Pathological findings}

All six patients underwent transbronchial biopsy (TBBx) during bronchoscopy at initial ICI pneumonitis presentation. Interestingly, as shown in figure 2, evidence of bronchiolitis obliterans with organizing pneumonia (BOOP) was found on all six pathological specimens. Tissue cultures were negative for infection in all six cases. No granulomas or lymphoid aggregates were observed in any of the pathological specimens.

As mentioned earlier, we previously showed that alveolar lymphocytosis was present in the BALF of patients with chronic ICI pneumonitis. To determine if similar lymphocytic infiltration was present in the lung parenchyma, we performed mIF to determine the cellular characteristics of BOOP observed on H\&E staining in the aforementioned samples. Interestingly, as shown in figure 3, mIF analysis also revealed significant lymphocytic infiltration, specifically of proliferative $\left(\mathrm{Ki} 67^{+}\right) \mathrm{PD}-1^{+}$
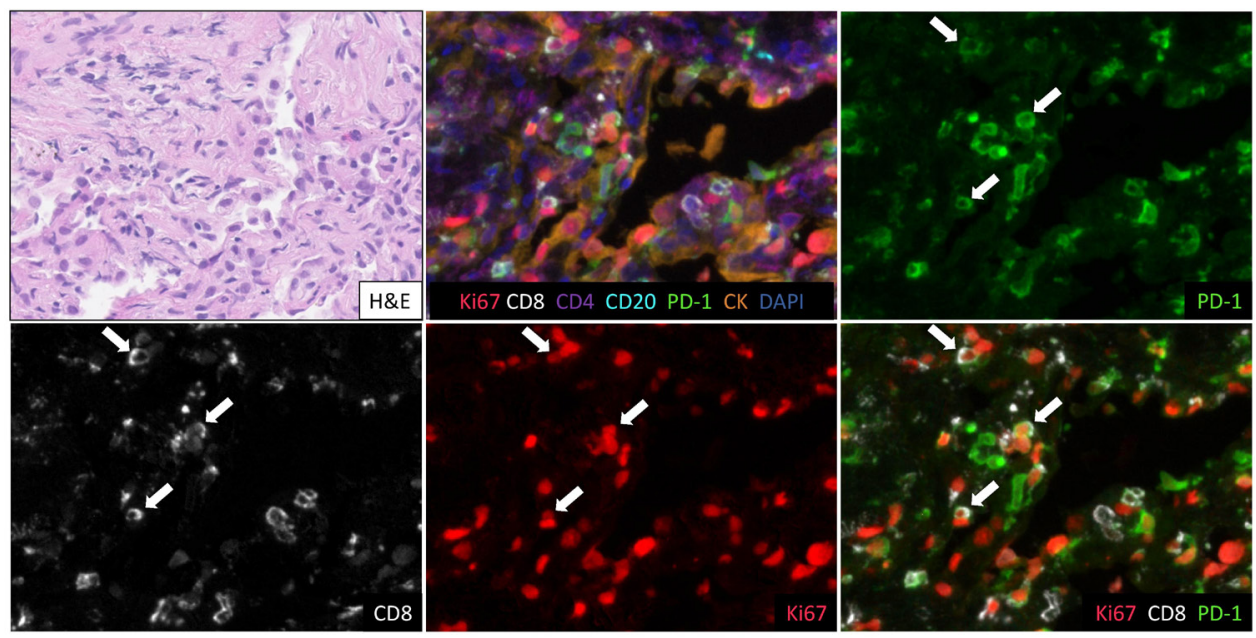

Figure 3 Highly proliferative PD $-1^{+}$cell accumulation characterizes chronic immune checkpoint inhibitor pneumonitis. Low power H\&E and immunofluorescence images highlight the relative abundance of Ki67 $7^{+} \mathrm{PD}-1^{+}$lymphocytes in chronic pneumonitis (top row) relative to sparse inflammatory cells seen in histologically unremarkable lung tissue (bottom row). Original magnification 200x. PD-1, programmed death 1. 
CD8 cells in lung biopsy samples at the time of pneumonitis re-emergence during steroid taper. To determine whether tissue lymphocytosis was a global phenomenon or restricted to the areas with histopathological evidence of BOOP, we examined tissue sections in another patient where areas of BOOP and adjacent normal lung were available. As shown in online supplementary figure 2, we

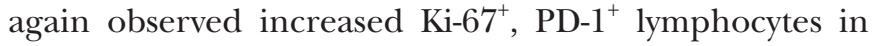
the areas affected by pneumonitis but such lymphocytic infiltration was not present in adjacent normal lung.

\section{Steroid therapy and additional immunosuppression}

Following diagnosis of ICI pneumonitis based on clinical, radiological (and pathological, when available) grounds, all patients were initiated on steroid treatment. As shown in online supplementary table 3, the initial steroid dosing was between $60 \mathrm{mg}$ and $120 \mathrm{mg}$ of prednisone (or equivalent). However, unlike typical cases of ICI pneumonitis, the median total duration of steroids in these six cases of chronic ICI pneumonitis was 37 weeks (rang: 16-43+ weeks). This is because radiographic and clinical evidence of ICI pneumonitis recrudesced when steroids were tapered to $10 \mathrm{mg}$ or less, and necessitated reinitiation of steroids followed by prolonged tapers in all six cases. Temporal changes in steroid dosing at the patient level and overall are outlined in online supplementary figure 3. In three out of six patients, ongoing steroid requirement necessitated initiation of second-line ICI pneumonitis therapy (either mycophenolate mofetil, infliximab or intravenousimmunoglobulin; table 1).

\section{DISCUSSION}

ICI-associated pneumonitis that persists or worsens with steroid tapering and necessitates $\geq 12$ weeks of immunosuppression after ICI discontinuation (ie, chronic ICI pneumonitis) has not been previously described. In one report of patients with melanoma with ICI pneumonitis, persistent radiographic evidence of pneumonitis was seen in $20 \%$ of cases. ${ }^{13}$ Whether a subset of these patients were also clinically symptomatic is not known. Here, we present a comprehensive clinical, radiographic and pathological assessment of this phenomenon across tumor types.

In our study, we report the incidence of chronic ICI pneumonitis at $2 \%$ in patients with both NSCLC and melanoma treated with anti-PD-(L) $1 \pm$ CTLA4 ICIs. We describe the clinical course of six patients with chronic ICI pneumonitis, and in these patients we note: (1) that the time to onset of chronic ICI pneumonitis is highly variable, (2) no relationship between onset of chronic ICI pneumonitis and other irAEs, (3) no worsening of ICI pneumonitis grade between initial and subsequent episodes, (4) median steroid durations well in excess of the recommended 4-6 weeks, (5) persistent BALF lymphocytosis despite steroid therapy and (6) histopathological evidence of BOOP with increased infiltration of highly proliferative $\mathrm{CD}^{+} \mathrm{T}$-cells.

From a management standpoint, ICI pneumonitis eventually improved/resolved in three patients, while three patients necessitated long-term, continuing steroid use. This is in contrast with currently published literature on irAEs from anti-PD(L) 1 therapy, which suggests that most toxicities, except for endocrinopathies and selected arthritides, resolve with a relatively short corticosteroid taper of 4-6 weeks. Prospective studies with longer follow-up periods are needed to determine the clinical course of pneumonitis in ICI-treated patients.

Fundamentally, this population of patients with chronic ICI pneumonitis likely represents a clinically distinct population different from patients with a singleton episode of acute ICI pneumonitis. For one, a significant survival bias exists; excluding the one patient who developed ICI pneumonitis at day 0 , five out of six patients developed pneumonitis only after at least 7 months of ICI therapy. This suggests that these patients experienced sufficient tumor responses to survive long enough to develop chronic ICI pneumonitis. When adjusted for guaranteed time bias, we recently showed that development of ICI pneumonitis actually worsened survival in NSCLC. ${ }^{14}$ However, given the lack of any patients with grade 4 pneumonitis and the prolonged time on ICI therapy before onset of symptoms in five out of six patients, the effect of chronic ICI pneumonitis on survival may be different, and similar to other irAEs, where a survival benefit has been observed. Our comparison of patients with chronic versus acute ICI pneumonitis suggests that the type of immunotherapy (eg, combination vs monotherapy) may be a risk factor for chronic ICI pneumonitis. However, given the small numbers involved, these intriguing findings will need to be replicated in a larger dataset.

Our pathology and bronchoalveolar lavage findings, taken together, suggest that the continued presence of proliferative lymphocytes, possibly T-cell subsets that are resistant to steroid-induced apoptosis, may be contributing to the pathobiology of chronic ICI pneumonitis. In this study, we observed lymphocytes in the BALF and tissue of patients with chronic ICI pneumonitis. This extends our earlier observations of increased T-cell subsets in the BALF of patients during the first episode of ICI pneumonitis, prior to initiation of steroids. ${ }^{12}$ Clinically, the presence of BALF lymphocytes, ongoing steroid requirement, and steroid dose (10-20 $\mathrm{mg}$ of prednisone/equivalent) at which ICI pneumonitis re-emerges is similar to the natural history of refractory cryptogenic organizing pneumonia (COP). Indeed, the overall duration and rate of steroid tapering presented in this study is similar to prior reports of rates of steroid weaning in $\mathrm{COP}^{15}$ Despite these initial observations, whether the presence of BOOP on TBBx is sufficient to increase the pretest suspicion for a patient developing chronic ICI pneumonitis remains unknown. In one other published series, a range of pathological appearances were seen at the time of acute ICI pneumonitis, including cellular interstitial pneumonitis, organizing pneumonia, diffuse alveolar damage, or no notable pathological findings. ${ }^{4}$ The early observations in this manuscript, however, provide a rationale for prospective study of BALF cell differentials and 
lung tissue cytometry in patients with ICI pneumonitis, to determine whether the pathological findings observed during the index ICI pneumonitis episode could serve as biomarkers for risk of subsequent re-emergence, or could inform the length of steroid tapering.

In conclusion, this report of chronic pneumonitis from anti-PD-(L) 1 therapy describes a unique subset of ICI pneumonitis with a distinct natural history. These patients require longer durations of corticosteroid therapy, and occasional lifelong immunosuppression. Lung biopsy data from this study suggest that the presence of organizing pneumonia on histological assessment may portend a chronic clinical course for ICI pneumonitis. Future areas of study include identifying whether this phenomenon may occur in other tumor types treated with ICIs, examining the relationship between BALF cell differential as a means to risk stratify outcomes for symptomatic ICI pneumonitis, and investigating associations between BALF, lung biopsy features, and both response to immunosuppression and clinical ICI pneumonitis outcome in a prospective fashion. Crucially, even in a small number of patients, these data suggest a role for bronchoscopic assessment where possible for patients with ICI pneumonitis, outside of ruling out alternative diagnoses-an area that has been hotly debated in the medical oncology field.

\section{Author affiliations}

${ }^{1}$ Oncology, Johns Hopkins Medicine Sidney Kimmel Comprehensive Cancer Center, Baltimore, Maryland, USA

${ }^{2}$ The Bloomberg Kimmel Institute for Cancer Immunotherapy, Johns Hopkins University, Baltimore, Maryland, USA

${ }^{3}$ Pathology, Queen's University, Kingston, Ontario, Canada

${ }^{4}$ Pathology, Johns Hopkins Medicine Sidney Kimmel Comprehensive Cancer Center, Baltimore, Maryland, USA

${ }^{5}$ Division of Pulmonary Critical Care Medicine, Johns Hopkins University, Baltimore, Maryland, USA

${ }^{6}$ Radiation Oncology, Johns Hopkins Medicine Sidney Kimmel Comprehensive Cancer Center, Baltimore, Maryland, USA

${ }^{7}$ Radiology, Johns Hopkins University, Baltimore, Maryland, USA

Twitter Jarushka Naidoo @DrJNaidoo and Joanne Riemer @jriemer3

Contributors JN, KS, EL, PMF, TC, PBI, LBY, DF-K, HL, JR, JRB, SKD and FRD: identification, analysis, and interpretation of patient data. TC, DW and JT: multispectral immunofluorescence examination and interpretation. PBI: histological examination lung biopsy samples. CTL and KS: radiological data analysis and interpretation. JN and KS: study design, conceptualization, and manuscript writing. All authors read and approved the final manuscript.

Funding Bloomberg-Kimmel Institute for Cancer Immunotherapy (JN). Funding was used to support multiplex immunofluorescence data collection, analysis and interpretation.

Competing interests JN: research funding: AstraZeneca, Merck, Consulting/ Advisory Board: AstraZeneca, Merck, Bristol-Myers Squibb, Honoraria: AstraZeneca, Merck, Bristol-Myers Squibb. EL: research funding: Bristol-Myers Squibb, Merck, Regeneron, Consulting/Advisory Board: Bristol-Myers Squibb, Merck, Novartis, EMD Serono, Array BioPharma, MacroGenics, Sanofi. PMF: research funding: AstraZeneca, Bristol-Myers Squibb, Corvus, Kyowa, Novartis, Consulting/Advisory Board: AstraZeneca, Bristol-Myers Squibb, Janssen, Merck, Novartis, Lilly, Boehringer. LBY: research funding: Rocket Medical, Consulting/Advisory Board: Boston Scientific. HL: Consulting/Advisory Board: Boston Scientific; DFK/Advisory Board: Consulting: Boston Scientific. JT: research funding: Bristol-Myers Squibb,
Consulting/Advisory Board: Bristol-Myers Squibb, Merck, AstraZeneca. JRB: research funding: Bristol-Myers Squibb, Consulting/Advisory Board: Bristol-Myers Squibb, Merck, AstraZeneca, and Genentech, and reports receiving commercial.

\section{Patient consent for publication Obtained.}

Ethics approval Ethical approval was obtained by the Johns Hopkins Hospital IRB for collection of clinical, radiographic and pathological data and patient samples and biospecimen collection protocols J1655 (PI: PMF) and J13139 (PI: EJL) as well as institutional database Z15114 (PI: JN).

Provenance and peer review Not commissioned; externally peer reviewed.

Data availability statement Data are available upon reasonable request. The datasets used during the current study are available from the corresponding author on reasonable request.

Open access This is an open access article distributed in accordance with the Creative Commons Attribution Non Commercial (CC BY-NC 4.0) license, which permits others to distribute, remix, adapt, build upon this work non-commercially, and license their derivative works on different terms, provided the original work is properly cited, appropriate credit is given, any changes made indicated, and the use is non-commercial. See http://creativecommons.org/licenses/by-nc/4.0/.

\section{ORCID iDs}

Jarushka Naidoo http://orcid.org/0000-0002-3470-8686

Evan J Lipson http://orcid.org/0000-0003-2976-0911

\section{REFERENCES}

1 Naidoo J, Page DB, Li BT, et al. Toxicities of the anti-PD-1 and antiPD-L1 immune checkpoint antibodies. Ann Oncol 2016;27:1362.

2 Nishino M, Sholl LM, Hatabu H, et al. Anti-PD-1-Related pneumonitis during cancer immunotherapy. N Engl J Med 2015;373:288-90.

3 Nishino M, Ramaiya NH, Awad MM, et al. PD-1 Inhibitor-related pneumonitis in advanced cancer patients: radiographic patterns and clinical course. Clin Cancer Res 2016;22:6051-60.

4 Naidoo J, Wang X, Woo KM, et al. Pneumonitis in patients treated with anti-programmed death-1/programmed death ligand 1 therapy. JCO 2017;35:709-17.

5 Sears CR, Peikert T, Possick JD, et al. Knowledge gaps and research priorities in immune checkpoint Inhibitor-related pneumonitis. An official American thoracic society research statement. Am J Respir Crit Care Med 2019;200:e31-43.

6 Brahmer JR, Lacchetti C, Thompson JA. Management of immunerelated adverse events in patients treated with immune checkpoint inhibitor therapy: American society of clinical oncology clinical practice guideline summary. J Oncol Pract 2018;14:247-9.

7 Puzanov I, Diab A, Abdallah K, et al. Managing toxicities associated with immune checkpoint inhibitors: consensus recommendations from the society for immunotherapy of cancer (SITC) toxicity management Working group. J Immunother Cancer 2017;5:95.

8 Thompson JA. New NCCN guidelines: recognition and management of immunotherapy-related toxicity. J Natl Compr Canc Netw 2018;16:594-6.

9 Topalian SL, Taube JM, Pardoll DM. Neoadjuvant checkpoint blockade for cancer immunotherapy. Science 2020;367:eaax0182.

10 Suresh K, Voong KR, Shankar B, et al. Pneumonitis in non-small cell lung cancer patients receiving immune checkpoint immunotherapy: incidence and risk factors. J Thorac Oncol 2018;13:1930-9.

11 Suresh K, Psoter KJ, Voong KR, et al. Impact of checkpoint inhibitor pneumonitis on survival in NSCLC patients receiving immune checkpoint immunotherapy. J Thorac Oncol 2019;14:494-502.

12 Suresh K, Naidoo J, Zhong Q, et al. The alveolar immune cell landscape is dysregulated in checkpoint inhibitor pneumonitis. $J$ Clin Invest 2019;130:4305-15.

13 Johnson DB, Taylor KB, Cohen JV, et al. Anti-PD-1-Induced pneumonitis is associated with persistent imaging abnormalities in melanoma patients. Cancer Immunol Res 2019;7:1755-9.

14 Suresh K, Naidoo J. Lower survival in patients who develop pneumonitis following immunotherapy for lung cancer. Clin Lung Cancer 2020;21:e169-70.

15 Lazor R, Vandevenne A, Pelletier A, et al. Cryptogenic organizing pneumonia. Characteristics of relapses in a series of 48 patients. The groupe d'etudes et de recherche sur les maladles "orphelines" pulmonaires (GERM"O"P). Am J Respir Crit Care Med 2000;162:571-7. 


\section{Correction: Chronic immune checkpoint}

\section{inhibitor pneumonitis}

Naidoo J, Cottrell TR, Lipson EJ, et al. Chronic immune checkpoint inhibitor pneumonitis. J Immunother Cancer 2020;8:e000840. doi: 10.1136/jitc-2020-000840.

Since the online publication of this article, the authors have noticed the following errors: Figure 2 is incorrect. Please see corrected figure 2 shown below as figure 1 :

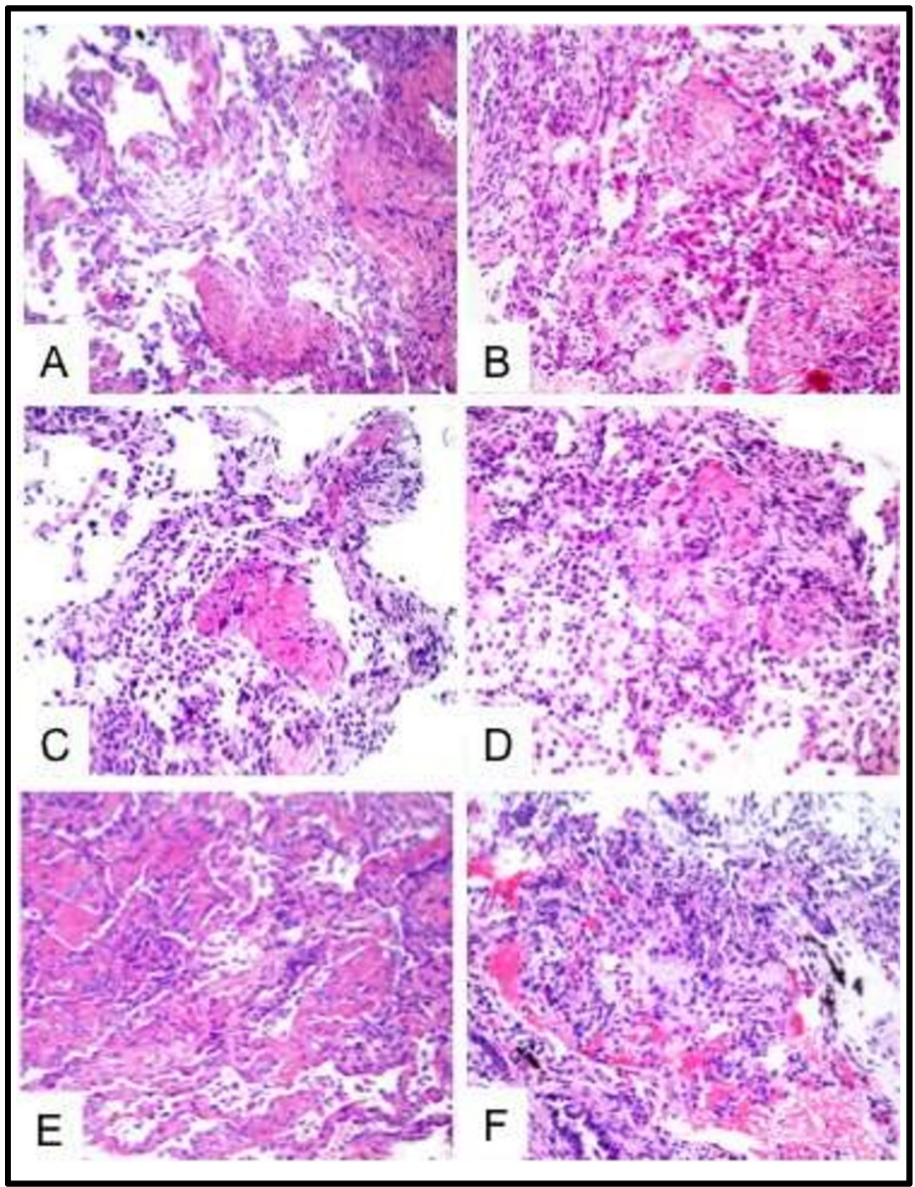

Figure 1 Pathologic Features of Patients with Chronic PD-1 Pneumonitis. H\&Estaining of lung biopsy samples from patients with chronic pneumonitis. (A)Patient 1: Bronchiolitis obliterans Organizing Pneumonia (BOOP), described inmanuscript as organizing pneumonia, with an intra-alveolar fibroblast plug andorganizing alveolar fibrin; (B) Patient 2: Acute lung injury with acutefibrinous organizing pneumonia and organizing diffuse alveolar damage; (C) Patient 3: Organizing alveolar fibrin consistent with exudative phase of BOOP;(D) Patient 4: Organizing pneumonia (BOOP) with organizing alveolar fibrin; (E)Patient 5: Organizing alveolar fibrin consistent with exudative phase of BOOP;(F): Patient 6: Organizing pneumonia (BOOP). 
The legend for figure 3 was incorrectly linked to online supplemental figure 2 and vice versa. The correct figure 3 legend is 'Chronic pneumonitis is associated with brisk lymphocytic inflammation, including many proliferating PD-1 +CD8+T cells. A representative case of chronic pneumonitis (Patient 5) with abundant lymphocytic inflammation is shown (H\&E, top left, see also figure 2). Profiling of the inflammatory microenvironment with multiplex immunofluorescence (top center) reveals a dramatic recruitment of PD-1 +lymphocytes (green, top right) and numerous CD8 +cytotoxic T cells (white, bottom left). Many of the PD-1 +and CD8+lymphocytes are positive for the proliferation marker Ki67 (red, bottom center), including numerous PD1 +CD8+T cells (bottom right, arrows highlight PD-1 +CD8+Ki67+cells). Original magnification $200 x$.'

The correct online supplemental figure 2 legend is 'Highly proliferative PD-1 +cell accumulation characterizes chronic pneumonitis. Low power H\&E and mIF images highlight the relative abundance of Ki67 +PD-1+lymphocytes in chronic pneumonitis (top row) relative to sparse inflammatory cells seen in histologically unremarkable lung tissue (bottom row). Original magnification 200x.'

The updated supplementary files are linked to this correction article.

Open access This is an open access article distributed in accordance with the Creative Commons Attribution Non Commercial (CC BY-NC 4.0) license, which permits others to distribute, remix, adapt, build upon this work non-commercially, and license their derivative works on different terms, provided the original work is properly cited, appropriate credit is given, any changes made indicated, and the use is non-commercial. See http://creativecommons.org/licenses/by-nc/4.0/.

(c) Author(s) (or their employer(s)) 2020. Re-use permitted under CC BY-NC. No commercial re-use. See rights and permissions. Published by BMJ.

J Immunother Cancer 2020;8:e000840corr1 . doi:10.1136/jitc-2020-000840corr1

Check for updates 